\title{
Numerical Simulation Techniques Research and Application in Genetic Algorithm Design
}

\author{
Yamian Peng ${ }^{*}$, Chunfeng Liu and Dianxuan Gong
}

Hebei United University, Tangshan Hebei 063000, China

\begin{abstract}
Numerical simulation techniques are also called computer simulation, which take the computer as a means to study all kinds of engineering and physical problems even natural objective through numerical calculation method and image display. This paper studied the numerical simulation techniques and try to solve two-dimensional convectiondiffusion equation parameter identification inverse problem by the genetic algorithm. Firstly, the finite element method was illustrated to solve the steady problem of two-dimensional convection-diffusion equation before it compute parameter identification inverse problem each time. Subsequently, it can search the best approximate solution from many initial points and obtained the global optimum solution by means of crossover operator and mutation operator. Finally, the paper discussed the computer simulation of GA for solving the inverse problem, and puts forward a new method for solving inverse problem: Genetic algorithm based on the best disturbed iteration. The results of numerical simulation show that the genetic algorithm has the higher accuracy and the quicker convergent speed. And it is easy to program and calculate and is of great application.
\end{abstract}

Keywords: Genetic algorithm, inverse problem, numerical simulation techniques, the best disturbed iteration.

\section{INTRODUCTION}

Development of numerical simulation technology was in numerical solution in the 60 's. Progress in the generation of numerical simulation is mainly in the crude technology, parallel computing, grid PEBI, in 90 years. Simulation technology development is reflected in two aspects for twenty-first century. One is the integration of simulation technology, on the other hand is quantitative analysis of attribute uncertainty and its effects on the calculation.

It is assumed that $\phi=\phi(x, y, z, t)$ is a kind of physical quantity carried by the fluid of unit volume. The physical quantity can be the pollutants present in the fluid or in the temperature of the fluid; it can also be the quality of the fluid medium itself. So $\phi$ can represent the concentration of polluting substances contained in the fluid or the temperature of the fluid, and it can also represent the density of the fluid $\rho$. $\mathrm{c}$ is the concentration of polluting substances or fluid temperature, which can also be the density of fluid. So it can be said that the physical $\phi$ is like this product of $\rho \phi$. In order to study the Changes in law of the physical, it removes a limited region $D$ freely in the flow. Its border is $S$. What it interests is the distribution and change of the physical $\phi$ in region $D$. The parameter identified problem is a kind of common inverse problem in many fields of natural science and engineering technology. There are many ways for the parameter identified inverse problem. Measuring the

*Address correspondence to this author at the Hebei United University, Tangshan Hebei 063000, China; Tel: 0086-315-2592281;

Fax: 0086-315-2592281; E-mail: yamianpeng@yeah.net parameter is the most easiest and direct method for the problem, but this method is difficult to analyze and the measurement is very expensive, etc. So, reason out the unknown parameters, people seek the values of unknown parameters.

To solve optimal inverse problems of partial differential equations of momentum in the process, one must first determine the unknown quantities at the true solution near the initial guess value. How to select the appropriate initial value is the best disturbed iteration for solving the key inverse problem. This paper will discuss the feasibility of GA for solving the inverse problem, and puts forward a new method for solving inverse problem: genetic algorithm based on the best disturbed iteration.

\section{FINITE ELEMENT METHOD}

Consider two-dimensional steady problem of convectiondiffusion equation (3):

$$
\left\{\begin{array}{lr}
u \frac{\partial c}{\partial x}+v \frac{\partial c}{\partial y}=\frac{\partial}{\partial x}\left(k \frac{\partial c}{\partial x}\right)+\frac{\partial}{\partial y}\left(k \frac{\partial c}{\partial y}\right)+a c+f & (x, y) \in \Omega \Omega \\
c(x, y)=f_{1}(x, y) & (x, y) \in \partial \Omega_{1} \\
\frac{\partial c}{\partial n}=f_{2}(x, y) & (x, y) \in \partial \Omega_{2}
\end{array}\right.
$$

The area $\Omega$ is a bound area on the $x O y$ surface. $\partial \Omega_{1}$ and $\partial \Omega_{2}$ are boundaries of $\Omega$ and $\partial \Omega_{1}+\partial \Omega_{2}=\partial \Omega$.

The inverse problem is to seek unknown parameter function $k(x, y)$ according to equations (1)-(3). Because the function $c(x, y)$ is also unknown, so it cannot solve $k(x, y)$ 
from equations (1)-(3). Then the additional condition as follows:

$c(x, y)=p(x, y),(x, y) \in \partial \Omega_{2} \subset \partial \Omega$

(1).

Geometric area of the inverse problem is shown in Fig.

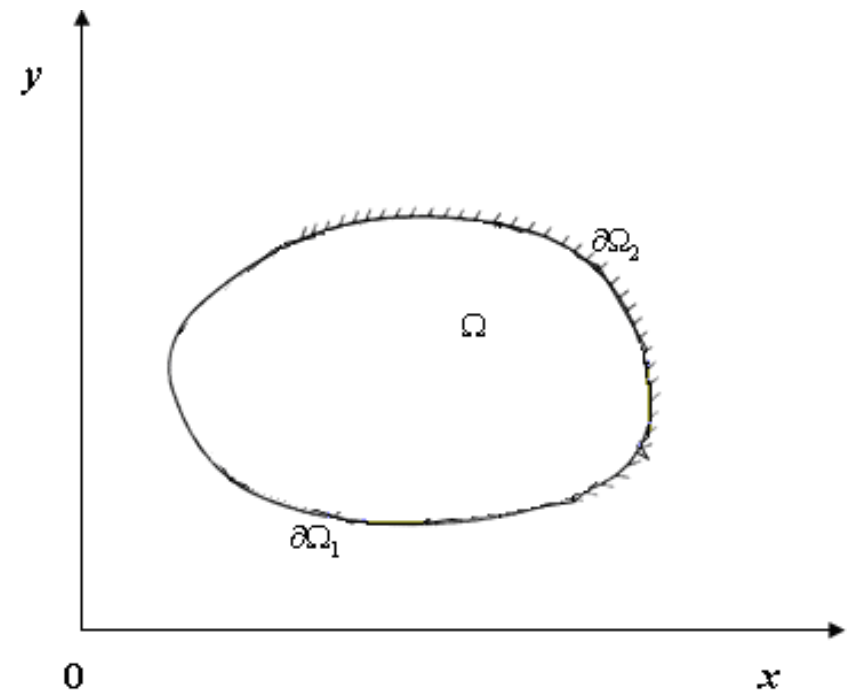

Fig. (1). Area of the inverse problem.

So, the inverse problem is to find $k(x, y)$ according to the known functions $u(x, y), v(x, y), \quad a(x, y), \quad f(x, y)$, $f_{1}(x, y), f_{2}(x, y)$ and additional condition $p(x, y)$. The function $p(x, y)$ can only get values in some measure points which are normally in practice. Suppose the points $\left(x_{i}, y_{j}\right)$, $i=1,2, \cdots, l, j=1,2, \cdots q$ on the boundary $\partial \Omega_{2}$ are know then the inverse problem is to find $k(x, y)$ according to the known functions and the values of $p(x, y)$ in the measure points [4].

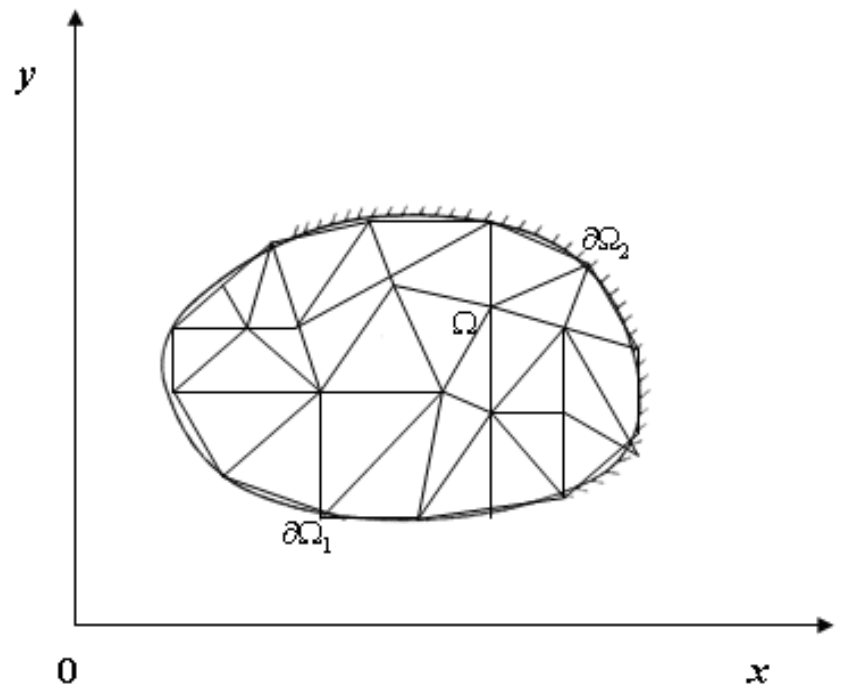

Fig. (2). The dissection of $\Omega$.

The approximate solution of inverse problem in every compute process is according to the approximate solution of the steady problem. So the high precise method of the steady problem must be studied [5]. In the process of compute the inverse problem, it is required to compute problem (1)-(3) every time before. The procedure steps of finite element method for problem (1)-(3) are as follows.

Step 1: Cut the area $\Omega$ to some non-Multifoods basic elements as triangular shape in this paper. The vertex of triangular shape can be named as $p_{i}$ and its coordinate number $\left(x_{i}, y_{i}\right)$ are assured numbers.

Step 2: Number fundamental elements and nubs must be in an order as fundamental elements inside prior to side ones and nubs inside prior to side ones.

Step 3: Constructed interpolating function that is the solution of equation (1) in every fundamental elements.

This way approximate solution of the problem (1)-(3) can be attained. In this proceed produce the first error of precise $k(x, y)$ and its approximate solution [6].

From mathematical angle, the paper expounds that although inverse problem have various types, they can be considered to be the category of operator theory. On the one hand this can solve inverse problem under a unified framework of operator theory, on the other hand the application of operator theory can lead to a deeper understanding of the essence of inverse problem and guide the solution approach, thus building a necessary math theoretical basis for inverse problem research.

Suppose $\left\{\phi_{i}(x, y)\right\}$ were the primary functions or orthogonal primary functions and let the form of $k(x, y)$ as follows:

$$
\begin{aligned}
& k(x, y)=\sum_{i=1}^{\infty} k_{i} \phi_{i}(x, y) \\
& \bar{k}_{n}(x, y)=\sum_{i=1}^{n} k_{i} \phi_{i}(x, y)
\end{aligned}
$$

Then

$$
k(x, y)=\lim _{n \rightarrow \infty} \bar{k}_{n}(x, y)=\lim _{h \rightarrow \infty} \sum_{i=1}^{n} k_{i} \phi_{i}(x, y) .
$$

For the suitable number $n$, it takes the addition $\bar{k}_{n}(x, y)$ of finite primary functions [3]. So this kind of inverse problem seeks $\bar{k}_{n}(x, y)$ according to known functions $u(x, y), v(x, y), a(x, y), \quad f(x, y), \quad f_{1}(x, y), \quad f_{2}(x, y)$ and the additional condition $p(x, y)$. Because primary functions were known so the problem of $\bar{k}_{n}(x, y)$ is to seeks an ndimensions vector $\left(k_{1}, k_{2}, \cdots, k_{n}\right)$ [4].

Suppose the solution of a steady problem (1) (3) that included $\bar{k}_{n}(x, y)$ is $c\left(k_{1}, k_{2}, \cdots, k_{n}, x, y\right)$, then the inverse problem inverts nonlinear optimization problem:

$\min _{\left(k_{1}, k_{2}, \cdots, k_{n}\right) \in R^{n}} \sum_{i=1}^{l} \sum_{j=1}^{q}\left(p\left(x_{i}, y_{j}\right)-C\left(k_{1}, k_{2}, \cdots, k_{n}, x_{i}, y_{j}\right)^{2}\right.$ 
Take formula (7) as adaptive function and adjust coefficients $k_{1}, k_{2}, \ldots ., k_{n}$ let value of formula (7) be minimum , then it can get the optimization approximating solution of $k(x, y)$ as follows:

$\bar{k}_{n}(x, y)=\sum_{i=1}^{n} k_{i} \phi_{i}(x, y)$

The choice of number $\mathrm{n}$ and orthogonal primary functions $\left\{\phi_{i}(x, y)\right\}$ will effect the precision of $k(x, y)$. But the genetic algorithm of this inverse problem can compute high precision solution of $k(x, y)$ with small $\mathrm{n}$, because it can search the good coefficients $k_{1}, k_{2}, \ldots ., k_{n}$ of $k(x, y)$.

\section{THE BEST DISTURBED ITERATION METHOD}

In environmental hydraulics, the mathematical models of many problems is the steady problem as follows:

$$
\left\{\begin{array}{ccc}
L[c(x), t] u(x, t)=0 & x \in \Omega & t>0 \\
B u(x, t)=f(x, t) & x \in \partial \Omega & t>0 \\
E u(x, 0)=g(x) & x \in \Omega &
\end{array}\right.
$$

Signal $u$ is a vector function and $L$ is a matrix differential operator and $B$ is a matrix edge condition operator and $E$ is a matrix initial operator and $c(x)$ is the unknown vector function. $L$ is linear depend on $c(x) . \Omega$ is the area. $\partial \Omega$ is the borderline of $\Omega$.

The inverse problem of this kind problem is to find the unknown vector function $c(x)$ of $L$ from the (1) and additional conditions as follows:

$$
\begin{aligned}
& \left.u(x, t)\right|_{\partial \Omega^{\prime}}=\phi(x, t) \quad x \in \partial \Omega^{\prime} \subset \partial \Omega \\
& \text { or }\left(\left.u(x, t)\right|_{t=T}=\phi(x) \quad x \in \Omega\right)
\end{aligned}
$$

This kind of inverse problem can convert a solution of nonlinear operator equation as follows:

$$
A[c(x)]=\phi(x, t) \text { or }(A[c(x)]=\phi(x))
$$

The inverse problem can convert the extreme value problem of nonlinear functional by regularization method:

$J_{\alpha}(c)=\|A(c]-\phi\|_{L^{2}\left(\partial \Omega^{\prime}\right) x[0, T]}^{2}+\alpha \Omega(c)$

$\alpha$ is the regularization factor. $\Omega$ is the steady functional on $L^{2}(\Omega)$. Then it can get the numerical solution of the inverse problem by numerical methods.

The kernel process of the best disturbed iteration method is as follows:

Step 1: establish iterate process as follows:

$c_{n+1}(x)=c_{n}(x)+\delta c_{n}(x)$

The disturbed iteration $\delta c_{n}(x)$ was identified by the nonlinear functional minimum problem as follows:

$$
J_{\alpha}\left(\delta \boldsymbol{c}_{n}\right)=\left\|A\left[\boldsymbol{c}_{n}+\delta \boldsymbol{c}_{n}\right]-\phi\right\|_{L^{2}\left(\partial \Omega^{\prime}\right) \times[0, T]}^{2}+\alpha D\left(\delta \boldsymbol{c}_{n}\right)
$$

Step 2: Discrete the minimum problem and solve the numerical solution of $\delta c_{n}(x)$ by linearization method that is solving partial minimum problem of nonlinear functional problem.

It takes space $C[0, L]$ as solution space of parameter $E(x)$ and takes a group of primary functions $\phi_{1}(x), \phi_{2}(x), \ldots, \phi_{n}(x), \ldots$. then

$E(x)=\sum_{i=1}^{\infty} k_{i} \varphi_{i}(x)$

Let

$\Phi(x)=\left(\phi_{1}(x), \phi_{2}(x), \ldots, \phi_{n}(x)\right)^{T}$,

$K=\left(k_{1}, k_{2}, \ldots, k_{n}\right)^{T} \in R^{n}$,

then

$E_{n}(x)=K^{T} \cdot \Phi(x)$

$E(x)=\operatorname{Lim}_{n \rightarrow \infty} E_{n}(x)=\operatorname{Lim}_{n \rightarrow \infty} K^{T} \cdot \Phi(x)$

Take finite sum to approach as $E(x)=\sum_{i=1}^{n} k_{i} \varphi_{i}(x)$. The choice of $\mathrm{n}$ is according to the precision requisition. When the primary function was choose, the problem of solve $E(x)$ is solve a n-dimension integral vector as follows:

$K=\left(k_{1}, k_{2}, \ldots, k_{n}\right)^{T}$

The solution $E(x)=\sum_{i=1}^{n} k_{i} \varphi_{i}(x)$ must satisfy the equation (I) and additional condition (4).

On the other hand, for every settled $E(x)$ there exist $c(x, t)$ and homologous $c(x, T)=\phi(x)$. A nonlinear operator $A$ exists from $E(x) \mathrm{t} \quad c(x, T)=\phi(x)$. The parameter identified inverse problem solves nonlinear operator equation as follows:

$A(E(x))=\phi(x)$

$E(x)$ is a point of function space of unknown dispersion coefficient $E(x)$ and $\phi(x)$ are points of data space of measurement. By and large, the operator $\mathrm{A}$ is nonlinear and ill-posed, that means if the $\phi(x)$ has a tiny change then the $E(x)$ will have a large change. The error of data is ineluctable in the practice problem, so the solution of inverse problem with (9) will have an error with real solution of $E(x)$. Now it solve inverse problem will solve with (9) by the disturbed iteration method and can get the nonlinear functional minimum problem as follows:

$$
J_{\alpha}[E(x)]=\|A(E(x))-\phi(x)\|^{2}+\alpha \Omega(E(x))
$$

$\alpha$ is regularization parameter. $\Omega$ is the steady function and it take the norm of space $\mathrm{L}^{2}[0, \mathrm{~L}]$. The process of the best disturbed iteration method is as follows: 
Step 1: It establish numerical iterate process through operator and identifies disturbed iteration method and linearization technology as follows:

$E_{j+1}(x)=E_{j}(x)+\delta E_{j}(x)$

The initial value $E_{0}(x)$ is the function around real solution $E^{*}(x)$ and $\delta E_{j}(x)$ which is disturbed iteration and is solved by the nonlinear optimization problem as follows :

$J_{\alpha}\left(\delta E_{j}(x)\right)=\left\|A\left(E_{j}(x)+\delta E_{j}(x)\right)-\phi(x)\right\|^{2}+\alpha \Omega\left(\delta E_{j}(x)\right)$

Step 2: It signed solution of $E(x)$ with equations (3) as $c(E(x), x, t)$. Now add $E_{j}(x)$ as a tiny disturbed iteration as follows:

$\delta E_{j}(x)=\sum_{i=1}^{n} \delta k_{i}^{j} \varphi_{j}(x)=\delta K_{j}^{T} \cdot \Phi(x)$

It signed solution of $E_{j}(x)+\delta E_{j}(x)$ with equations (3) as $c\left(E_{j}(x)+\delta E_{j}(x), x, t\right)$. The problem of identified $\delta E_{j}(x)$ can convert and seek $\delta K_{j}=\left(\delta k_{1}^{j}, \delta k_{2}^{j}, \ldots, \delta k_{n}^{j}\right)$ and $\delta K_{j}$ which can be solved by partial minimum problem of target function as follows:

$$
F\left(\delta K_{j}\right)=\left\|c\left(E_{j}(x)+\delta E_{j}(x), x, T\right)-\phi(x)\right\|^{2}+\alpha \Omega\left(\delta K_{j}\right)
$$

Because $\delta E_{j}(x)$ is a tiny disturbed iteration and can be obtained from multivariate Taylor explosion as follows:

$$
\begin{aligned}
& c\left(E_{j}(x)+\delta E_{j}(x), x, t\right) \\
= & c\left(E_{j}(x), x, t\right)+\nabla_{k_{i}}^{T} c\left(E_{j}(x), x, T\right) \cdot \delta K_{j}+o\left(\left\|\delta E_{j}(x)\right\|\right)
\end{aligned}
$$

Then

$$
\begin{aligned}
F\left(\delta K_{j}\right) & =\left\|c\left(E_{j}(x), x, T\right)-\phi(x)+\nabla_{k_{i}}{ }^{T} c\left(E_{j}(x), x, T\right) \cdot \delta K_{j}\right\|^{2} \\
& +\alpha \Omega\left(\delta K_{j}\right)
\end{aligned}
$$

If there is an $M$ discrete point $x_{m},(m=1,2, \ldots, M)$ in interval $[0, L]$ and take $\Omega\left(\delta K_{j}\right)=\delta K_{j}^{T} \cdot \delta K_{j}$. Then

$$
\begin{aligned}
F\left(\delta K_{j}\right)= & \sum_{m=1}^{M}\left[c\left(E_{j}(x), x_{m}, T\right)-\phi\left(x_{m}\right)+\nabla_{k_{i}}{ }^{T} c\left(E_{j}(x), x_{m}, T\right) \cdot \delta K_{j}\right]^{2} \\
& +\alpha \delta K_{j}{ }^{T} \cdot \delta K_{j}
\end{aligned}
$$

Let

$$
\begin{gathered}
U=\left[\begin{array}{c}
c\left(E_{j}(x), x_{1}, T\right) \\
c\left(E_{j}(x), x_{2}, T\right) \\
\vdots \\
c\left(E_{j}(x), x_{m}, T\right)
\end{array}\right], U^{*}=\left[\begin{array}{c}
\phi\left(x_{1}\right) \\
\phi\left(x_{2}\right) \\
\vdots \\
\phi\left(x_{m}\right)
\end{array}\right], \\
A=\left(a_{m i}\right)_{M \times n}, a_{m i}=\frac{\partial}{\partial k_{i}} c\left(E_{j}(x), x_{m}, T\right)
\end{gathered}
$$

Then the last equality can simplify as follows:

$$
\begin{aligned}
F\left(\delta K_{j}\right)= & \delta K_{j}^{T} A^{T} A \delta K_{j}+2 \delta K_{j}^{T} \cdot A^{T}\left(U-U^{*}\right) \\
& +\left(U-U^{*}\right)^{T} \cdot\left(U-U^{*}\right)+\alpha \delta K_{j}^{T} \cdot \delta K_{j}
\end{aligned}
$$

The partial minimum $\delta K_{j}$ of $F\left(\delta K_{j}\right)$ satisfied linear equations as follows:

$\left(A^{T} A+\alpha I\right) \delta K_{j}=A^{T}\left(U^{*}-U\right)$

The equality (12) is Euler equation of functional (11). It take the solution of (12) into equality (8), then it can obtain disturbed iteration and finish an iterate circular.

Step 3: How to identify factor $a_{m i}$ in matrix $A$ ? The calculation formula of $a_{m i}$ can be obtained by numerical differential as follows:

$$
\begin{aligned}
& a_{m i}=\frac{\partial}{\partial k_{i}} c\left(E_{j}(x), x_{m}, T\right) \\
& =\frac{1}{\tau}\left[c\left(E_{j}(x)+\tau \varphi_{i}(x), x_{m}, T\right)-c\left(E_{j}(x), x_{m}, T\right)\right]
\end{aligned}
$$

There is a need to solve $E_{j}(x), E_{j}(x)+\tau \phi_{i}(x),(i=1,2, \ldots, n)$ counterpart steady problem (3) and get numerical solutions $c\left(E_{j}(x), x_{m}, T\right)$ and $c\left(E_{j}(x)+\tau \phi_{i}(x), x_{m}, T\right)$ first.

\section{GA SOLVEPARAMETER INVERSE PROBLEM}

With the fixed population size, GA only uses fitness value to guide searching and regenerate population through executing genetic operations such as selection, crossover and mutation with probability. GA can simulate biological evolution by computer technology. Suppose optimal problem is function minimization and the target function is $\min f: R^{n} \rightarrow R, f$. The fitness function is $F: I \rightarrow R$ and $I=\left\{a_{i} \mid i=1,2, \cdots, n\right\}$ is a space of individual $a_{i}$. The target variable set in $R^{n}$ is $x=\left\{x_{1}, x_{2}, \cdots, x_{n}\right\}$ and $\left[u_{i}, v_{i}\right]$ is the range of amount $x_{i}$. The scale of father generation is $n p \geq 1$ and the filial generation is $n c \geq 1$. It is an individual number through cross and variation in every generation. The community is $P(t)=\left\{a_{1}(t), a_{2}(t), \cdots, a_{n p}(t)\right\}$ in the 10th evolve iteration. The cross operator is $C: I^{n P} \rightarrow I^{n c}$ and its control parameter is cross probability $P_{c}$. The variation operator is $M: I^{n c} \rightarrow I^{n c}$ and its control parameter is variation probability $P_{m}$. The selection operator for the father generation is $S:\left(I^{n c} \cup I^{n p}\right) \rightarrow I^{n p}$ and its control parameter is selection probability $P_{s} . C, M, S$ are macroopertator and change from community to community.

The algorithm is as follows:

Step 1: Take Binary digit string simulated chromosome and one of the string simulated gene.

Step 2: Bring the target function or its transformation as fitness function to simulate the individual adaptation for living environment. 
Step 3: The cross of father generation. Supposed cross probability is $p_{c}$ and the current father community have $n p \cdot p_{c}$ individuals that were crossing.

Step 4: The filial string variation.

Step 5: Evolution iteration and turn to run step 2 until it get the fit solution or run the evolve iteration time. It takes the fitness highest string as optimal solution.

GA is a new optimal method between steady optimal method and random optimal method.

If we take the formula (7) as adaptive function in genetic algorithm and the parameter function $k(x, y)$ is approached by $\alpha_{0}+\alpha_{1} x+\alpha_{2} y$, the steps of genetic algorithm procedure would be as follows.

Procedure: General Genetic Algorithm

Begin

Random initialize the genus group, $\mathrm{P}(0), \mathrm{t}=0$

Compute the adaptive value of individual in $\mathrm{P}(0)$

Keep the best individual

While (not satisfied stop condition) do

Execute cross operator

Execute mutate operate

Compute the adaptive value of individual in $\mathrm{p}(\mathrm{t}+1)$, $\mathrm{t}=\mathrm{t}+1$

Replace the current individual with the last individual when the current individual is not better than the last one.

end

end

The function $\Phi(x)=\left(\phi_{1}(x), \phi_{2}(x), \ldots \ldots, \phi_{n}(x)\right)^{T}$ is used and the combination is taken as follows:

$c^{*}(x)=\sum_{i=1}^{\infty} k_{i}^{*} \varphi_{i}(x)$

As the simulated solution of unknown parameter $q=q(x)$, then through iteration method to seek for coefficients $k^{T}=\left(k_{1}, k_{2}, \ldots, k_{n}\right) \in R^{n}$ in the combination and hope to get the most approximate solution that most nearly the precise solution of $q=q(x)$. So the question is how to give appropriate original coefficients of $k^{T}=\left(k_{1}, k_{2}, \ldots, k_{n}\right) \in R^{n}$ to get the best approximate solution of $q=q(x)$. The strategy is through the GA to get appropriate original coefficients of $k^{T}=\left(k_{1}, k_{2}, \ldots, k_{n}\right) \in R^{n}$.

Obviously, that is $V_{i} \geq 0$ and $L_{h} V_{i}=K, i \in G_{h}$ when $i \in G_{h}$. Take $K=\max \left|R_{i}\right|$, then as follows:

$\max \left|e_{i}\right|=\max \left|u(i)-u_{i}\right| \leq \max V_{i} \leq \frac{\left(\left|b_{1}\right|+\left|b_{2}\right|\right)^{2}}{4 c \cdot\left(b_{1}{ }^{2}+b_{2}^{2}\right)} \max _{i \in G_{h}}\left|R_{i}\right|$
When differential equations satisfy the conditions, the coefficient matrix from equations derived is strictly diagonally dominant matrices. Then solutions to relatively simple equations and the most convenient are to use Gauss elimination method or use Croat method to solve them. When the matrix is diagonal dominance, the coefficient matrix of equations is not so good properties and may even be a singular matrix.

\section{SIMULATION RESEARCH}

Consider the inverse problem of hyperbolic equation parameter identified as follows:

$p(x) \frac{\partial^{2} h}{\partial t^{2}}=\frac{\partial}{\partial x}\left(q(x) \frac{\partial h}{\partial x}\right)+c(x, t) \frac{\partial h}{\partial x}+d(x, t) h+f(x, t)$,

$0<x<1,0<t<0.5$

$p(x)=1+x, c(x, t)=x+t, d(x, t)=x-t$,

$f(x, t)=-\left(x^{2}+3 x\right) e^{x+t}$,

The boundary conditions are:

$\left.h\right|_{x=0}=g_{1}(t)=e^{t},\left.h\right|_{x=1}=g_{2}(t)=e^{1+t}$,

$\left.h\right|_{t=0}=f_{1}(x)=e^{x},\left.\frac{\partial h}{\partial t}\right|_{t=0}=f_{2}(x)=e^{x}$,

The add-conditions are:

$h\left(x_{i}, 0.5\right)(i=0,1,2, \ldots N), x_{i}=i h, h=\frac{1}{N}, \quad N=10$.

The system of primary functions $\left\{\varphi_{i}(x)\right\}$ chooses as linear piecewise functions:

$\phi_{0}(x)= \begin{cases}1-n x, & x \in[0,1 / n], \\ 0, & \text { others }\end{cases}$
$\phi_{i}(x)=\left\{\begin{array}{ll}n\left(x-\frac{i-1}{n}\right), & x \in\left[\frac{i-1}{n}, \frac{i}{n}\right] \\ n\left(\frac{i+1}{n}-x\right), & x \in\left[\frac{i}{n}, \frac{i+1}{n}\right] \\ 0, & \text { others }\end{array}\right.$,
$\phi_{n}(x)= \begin{cases}n\left(x-\frac{n-1}{n}\right), & x \in\left[\frac{n-1}{n}, 1\right] \\ 0, & \text { others }\end{cases}$

Take $n=10$, the parameter $q=q(x)$ precise solution adopt as:

$q(x)=2 \phi_{0}(x)+2 \phi_{1}(x)+\phi_{2}(x)+\phi_{3}(x)+0.5 \phi_{4}(x)+0 \phi_{5}(x)$

$+0.5 \phi_{6}(x)+\phi_{7}(x)+\phi_{8}(x)+2 \phi_{9}(x)+2 \phi_{10}(x)$

Take the original parameter as $q_{0}(x)=\sum_{i=0}^{10} k_{i}^{0} \phi_{i}(x)$ and $k_{0}^{0}, k_{1}^{0}, \ldots \ldots, k_{10}^{0}$ are global variations in search interval to search the values approach the precise solution. The number 
of variations is taken as nvars $=11$, the size of group is taken as psize $=20$, the maximum evolution number taken as maxgen $=7000$, the cross probability is taken as $\mathrm{pc}=0.8$, the variance probability is taken as $\mathrm{pm}=0.2$, and used the GA seek solution was as follows:

$$
\begin{aligned}
& q(x)=2.177 \phi_{0}(x)+2.209 \phi_{1}(x)+1.565 \phi_{2}(x) \\
& +1.155 \phi_{3}(x)+1.161 \phi_{4}(x)-0.361 \phi_{5}(x)+1.073 \phi_{6}(x) \\
& +1.105 \phi_{7}(x)+1.024 \phi_{8}(x)+2.470 \phi_{9}(x)+1.712 \phi_{10}(x)
\end{aligned}
$$

The above solution is taken as original values at the beginning of the best disturbed iteration method. When the iteration time is $n=1000$, it can obtain the parameter solution and the approximate solution error is obtained as

$$
e=\frac{\left\|q-q_{1000}\right\|_{2}}{n}=8.17497 \times 10-3 \text {. }
$$

The precise solution and the approximate solution of $q=q(x)$ are shown in Fig. (3).

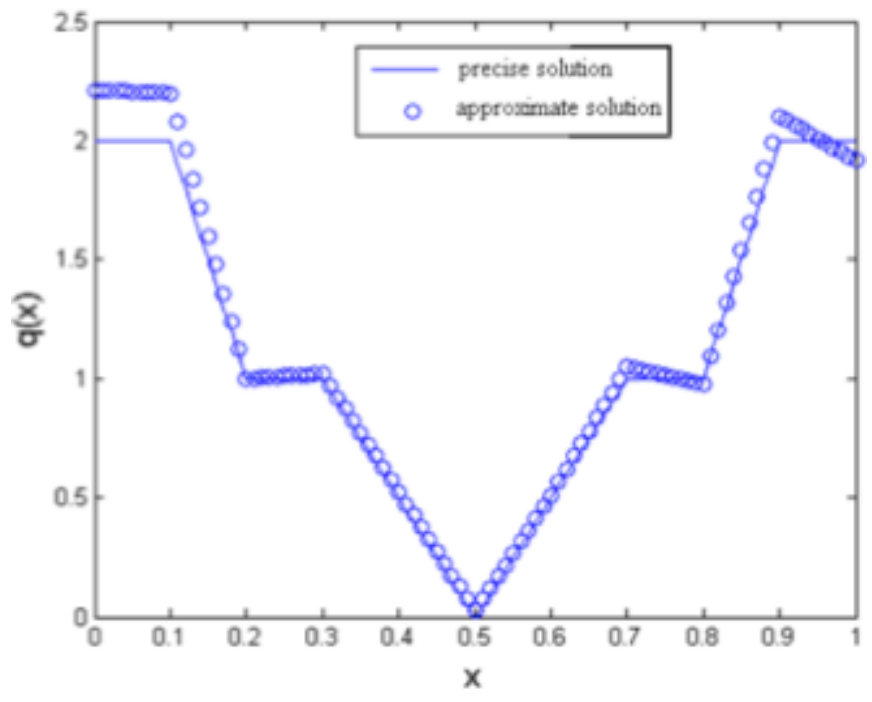

Fig. (3). the real values and approach value by 20 time iterates.

\section{CONCLUSIONS}

Numerical simulation in fact should be understood to use the computer in order to do the experiment. The realization of a particular computation in the computer is very similar to the performance of a physics experiment where the analyst has jumped out of the equation of circle to treat the physical phenomena, as an experiment in physics. The problem of parameter identification is a kind of inverse problem of partial differential equation and is applied broadly. The best disturbed iteration method is a classical method for inverse problem solving and the method has virtues as high stability and good astringency, but how to obtain the original values for iteration is a hard work. The numerical simulation outcome showed that the solution has good astringency and high precision.

\section{CONFLICT OF INTEREST}

The authors confirm that this article content has no conflict of interest.

\section{ACKNOWLEDGEMENTS}

This paper is supported by the Natural Scientific Fund Project in China (61170317), Natural Scientific Fund Project in Hebei Province (A2011209019), Hebei United University science study imbrues item (z201019).

\section{REFERENCES}

[1] L. Lin, L. Tao, and J. Tao, "Research on sample-level parallel simulation based on multi-node Multi-core environment, Journal of System Simulation, vol. 22, pp.115-119, 2011.

[2] Y. Wang, D. Wang, G. Liu, and Z. Yang, "SVC method for textile weave recognizing", JDCTA: International Journal of Digital Content Technology and its Applications, vol. 9, pp. 16-19, 2010.

[3] T.T.M. Onyango, D.B. Ingham, and D. Lesnic, "Reconstruction of heat transfer coefficients using the boundary element method", Computers \& Mathematics with Applications, vol. 56, pp.114-126, 2008.

[4] A. Greasley, "The case for the organisational use of simulation", Journal of Manufacturing Technology Management, vol. 15, pp. $560-566,2004$.

[5] F.U. Xiao-li, L.I. Da-mei, and J.I.N. Guo-yu, "Calculation of flowfield and analysis of spawning sites for Chinese sturgeon in the downstream of gezhoubadam", Journal of Hy-drodynamics, Ser. B, vol. 19, pp.78-83, 2007.

[6] N. Zabaras and B. Ganapathysubramanian, "A scalable framework for the solution of stochastic inverse problems using a sparse grid collocation approach". J. Computational Physics, pp. 4697-4735, 2008.

[7] F. Ternat, O. Orellana, and P. Daripa, "Two stable methods with numerical experiments for solving the backward heat equation", Applied Numerical Mathematics, vol. 61, pp. 266-284, 2011.

[8] P.T. Nam, D.D. Trong, and N.H. Tuan, "The truncation method for a two-dimensional nonhomogeneous backward heat problem", Applied Mathematics and Computation, vol. 216, pp. 3423-3432, 2010.

[9] V. Isakov, "Inverse problems for partial differential equations, volume 127 of Applied Mathematical Sciences. Springer Science Business Media, New York, 2006. 